\title{
Actitud ante el sida en estudiantes de enfermería. ¿Cuál es el papel de la formación académica?
}

\author{
J oaquín Tomás Sábado y Amor Aradilla Herrero \\ Escola Universitària d'I nfermeria Gimbernat. Sant Cugat del Vallès (Barcelona)
}

Los objetivos de est e estudio fueron conocer la actitud ante el SIDA que presentan los estudiantes de una escuela de enfermería $(N=242)$ y comprobar si esta actitud presenta variaciones significativas entre los diferentes cursos. Para la medición de la actitud se utilizó la Escala de Actitud ante el SIDA para Enfermería (EASE). Los resultados del ANOVA no revelaron diferencias estadísticamente significativas entre los tres cursos $(F=0.886 ; p=0,414)$. No obstante, a partir del análisis pormenorizado de las respuestas a los diferentes ítems de la escala se ponen de manifiesto opiniones que reflejan actitudes negativas $y$ prejuiciosas. Los resultados obtenidos plantean la necesidad de una reflexión profunda acerca de l as estrategias docent es más adecuadas para modificar positivamente las actitudes $y$ creencias que presentan los estudiantes ante el VIH/SIDA, dentro del objetivo general de formar profesionales capaces de dispensar cuidados de la máxima calidad, independientemente de la enfermedad o características personales de las personas a las que cuida.

Palabras clave: VIH-SIDA, actitudes, enfermería.
Attitude towards aids in nursing students. What is the role of academic formation?

The aims of this study were to know the attitude towards AIDS of the students of a nursing school ( $N=242)$ and check if this attitude presents significant variations between the different courses. The Nursing Scale of Attitude towards AIDS (EASE, Escala de Actitud ante el SIDA para Enfermería) was used to assess their attitude. The results of the ANOVA did not reveal statistically significant differences among the three courses $(F=0.886 ; p=0.414)$. Nevertheless, a detailed analysis of the answers to several items indicates that there are some negative and biased attitudes. The results obtained reveal the necessity of deep reflections about the most adequate educational strateg ies in order to improve the attitudes and believes of nursing students towards HIV/AIDS. The main aim is the formation of professionals who are able to give high quality care, independently of the patient's condition or personal characteristics.

Key Words: HIV/AIDS, Attitudes, Nursing

\section{INTRODUCCIÓN}

Correspondencia:

J oaquín Tomás Sábado

E scola Universitària d'I nfermeria Gimbernat

Vial Interpolar del Vallès, s.n.

08190 Sant Cugat del Vallès (Barcelona)

Telf.: 935893727 - Fax: 935891466

E-mail: joaquin.tomas@cesc.es
Veinte años después de su aparición, el Síndrome de Inmunodeficiencia Adquirida (SIDA) sigue constituyendo un grave problema de salud pública con importantes repercusiones sociales, que abarcan prácticamente todos los ámbitos: culturales, económicos, políticos, éticos y jurídicos. A lo largo de la historia, ninguna enfermedad, excepto, tal vez, la lepra, la peste o el trastorno mental, ha supuesto una estigmatización tan marcada de los afectados 
como el SIDA ${ }^{1}$. Las consecuencias de la enfermedad, su rápida extensión y sus características pandémicas han provocado la consideración del SIDA como la "plaga del siglo XX". Se ha dicho que el SIDA ha sido la enfermedad que más ha influido en el cambio de los hábitos sexuales de la sociedad actual, pero, además, su aparición ha transformado radicalmente muchos de los aspectos de la práctica de la medicina, enfermería y actividades asociadas, provocando un replanteamiento de cuestiones fundamentales de la función sanitaria. La cuestión de cómo proporcionar los cuidados adecuados a estos pacientes ha supuesto un importante aumento de las preocupaciones prácticas y éticas², 3 .

En un principio, el SIDA se asoció a la drogadicción y la homosexualidad, lo que favoreció un aumento de la transmisión heterosexual. En esta primera época se habla de grupos de riesgo (drogadictos, homosexuales, hemofílicos) descartando prácticamente la posibilidad de contagio en aquellas personas que no perteneciesen a uno de estos grupos. La poco afortunada denominación de grupos de riesgo ha sido sustituida en la actualidad por la más adecuada de conductas de riesgo, aunque algunos expertos consideran más adecuado hablar de prácti cas de riesgo.

EI SIDA presenta unas connotaciones muy especiales. Tradicional mente asociado al colectivo homosexual y a los usuarios de drogas por vía parenteral, durante más de una década ha sido considerado como la "plaga gay" y "enfermedad de drogadictos". La enfermedad, incluso ahora, es vista por algunos como un "castigo divino" hacia las que se consideran conductas sociales inmorales. Tales actitudes tienen una influencia sobre la opinión pública y sobre las reacciones de los profesionales de la salud hacia los dientes con SIDA. Se ha comprobado que estos profesionales presentan reacciones fóbicas relacionadas con el tratamiento del SIDA, que se identifican con homofobia, fobia a la muerte y fobia al contagio ocupacional ${ }^{4}$. De hecho, son numerosos los estudios que han mostrado que un número importante de enfermeras, médicos y otros profesionales de la salud presentan actitudes marcadamente negativas hacia las personas con SIDA que originan barreras que afectan a la calidad de los cuidados 5 .

El miedo al contagio es identifi cado como la prindpal fuente de reacciones negativas hacia las personas con SIDA en el personal sanitario ${ }^{6}$. No obstante, el riesgo de contraer el SIDA por parte de los profesionales sanitarios, que, en realidad, es decididamente bajo, seha magnificado, no sólo por la alta mor talidad que en sus inicios provocó, sino también por la percepción social de la enfermedad, que genera prejuicios sociales y mi edo irracional ${ }^{7}$, lo que, inevitablemente, se traducirá en actitudes negativas hacia la enfermedad y, sobretodo, hadia sus afectados.

Sin embargo, el miedo al contagio no es la única causa de la actitud negativa hacia los enfermos de SIDA. También la vía de contagio parece ser un factor importante en cuanto a las actitudes del personal sanitario. Cole ${ }^{8}$ encuentra que las actitudes con respecto a seropositivos en una muestra de 125 estudiantes de enfermería iban de más a menos positivas según el contagio hubiese sido por transfusión sanguínea, actividad heterosexual, actividad homosexual y por compartir agujas. También con estudiantes de enfermería, West et al. ${ }^{9}$ llegan a resultados similares, encontrando que los más estigmatizados son los contagiados por actividades relacionadas con el uso de drogas y contacto sexual, y los menos por transmisión materna o transfusión de sangre. La vía de contagio, y, en consecuencia, el estilo de vida, se manifiesta también como condicionante de la actitud en estudios realizados con profesionales de enfermería ${ }^{10}$.

Otras variables que se han identificado como moduladoras de la actitud hacia el SIDA son la ideología, la edad ${ }^{11}$, el estrés laboral ${ }^{12,13}$, actitudes sobre la homosexualidad ${ }^{14} \mathrm{y}$, en especial, las lagunas de conocimientos precisos sobre las características de la enfermedad y mecanismos de transmisión ${ }^{15}$ y la falta de experiencia clínica con pacientes con SIDA ${ }^{16}$.

En la numerosa literatura aparecida sobre la infección por el virus de la inmunodeficiencia humana $(\mathrm{VIH})$ y enfermería ${ }^{17}$, destacan dos temas principales: las actitudes negativas y ansiedades de las enfermeras relacionadas con el SIDA ${ }^{18,19}$ y la necesidad imperiosa de una educación efectiva para cambiar estas actitudes y reforzar los conocimientos y habilidades de las enfermeras ${ }^{20}$, ante la evidencia de que la educación tiene un papel decisivo en el cambio de actitudes ${ }^{21}$.

En el ámbito sanitario, es particularmente importante la actitud hacia los enfermos deSIDA en el caso del profesional de enfermería, como responsable del cuidado, tratamiento, vigilancia y aplicación completa de los cuidados necesarios, que implican, además, la utilizadión continua de técnicas invasivas que suponen contacto directo con la sangrey dros fluidos corporales, vehículos de transmisión de la enfermedad ${ }^{22}$. Parece evidente que una herramienta importante para evitar las actitudes negativas de las enfer meras 
hacia el SIDA será una formación adecuada, que debe comenzar en las Escuelas de Enfermería y debe capacitar al estudiante en todos los aspectos relacionados con la infección VIH y las formas de profección de la exposición, sin olvidar las cuestiones éticas y morales relacionadas ${ }^{23-25}$. Algunos investigadores sugieren incorporar intervenciones efectivas en las estrategias de educación, como psicodrama, revisiones personales y la colaboración de personas con SIDA en las sesiones educativas ${ }^{26}$, ya que se ha comprobado que las estudiantes de enfermería que realizan sus práciicas en unidades donde hay pacientes con SIDA presentan posteriormente una mejora en su actitud hacia el SIDA en general y en la intención de trabajar con pacientes seropositivos ${ }^{27}$.

El objetivo de este trabajo es conocer la actitud ante el SIDA que presentan los estudiantes de una Escuela de Enfermería y comprobar si esta actitud presenta variaciones significativas entre los diferentes cursos, utilizando como criterio las respuestas de los estudiantes a la Escala de Actitud ante el SIDA para Enfermería (EASE) ${ }^{28}$.

\section{MA TERIAL Y MÉTODO}

\section{Sujetos}

El estudio se realizó en la E scuela Universi taria de Enfermería Gimbernat, adscrita a la U niversidad Autónoma de Barcelona, duranteel pri mer trimestre del curso 2002-03, tomándose como población de estudio todos los alumnos matriculados en los tres cur sos en turno de mañana, en total 242 , de los cuales 215 eran mujeres y 27 hombres.

\section{Instrumento}

La recogida de datos se efectuó mediante la Escala de Actitud ante el SIDA para Enfermería (EASE), construida y validada por Tomás-Sábado ${ }^{28}$. La EASE es una escala tipo Likert de 21 ítems con cinco opciones de respuesta cada uno de ellos. Estas opciones van desde el total acuerdo al total desacuerdo con el reactivo propuesto. La puntuación asignada a cada ítem, en función de su direccionalidad es de 5 a 1 ( 5 para el total acuerdo y 1 para el total desacuerdo) para los ítems $\mathrm{N} 03,5,7,8,11,14$, 15 y 21 ; y de 1 a 5 ( 1 para el total acuerdo y 5 para el total desacuerdo) para los ítems № 1, 2, 4, 6, 9, 10, $12,13,16,17,18,19$ y 20 . Los dos extremos de puntuación de la escala están constituidos por una puntuación máxima de 105, que indica las actitudes más positivas; y una puntuación mínima de 21 , que indica las actitudes más negativas y prejuiciosas.
En Anexo figuran los enunciados de los 21 ítems de la EASE.

\section{Procedimiento}

El estudio se ajustó a un diseño observacional, descriptivo y transversal. Los cuestionarios se administraron colectivamente a los estudiantes por los propios profesores en el transaurso de su hor ario docente. Previamente se soli a tó su colaboración vol untaria, garantizando la confidencialidad y el anoni mato. Los datos se tabularon y analizaron mediante el paquete estadísti co SPSS 11.0 para Windowns, calculándose estadísti cos descriptivos, porcentajes y análisis de la varianza (ANOVA).

\section{RESULTADOS}

De los 242 alumnos matriculados respondieron a la escala un total de 205, 184 mujeres y 21 hombres, con una edad media de 22,06 años (DT $=4,41$ ) y un rango de 17 a 51 años. En cuanto a la distribución por cursos, 73 alumnos pertenecían a primer curso, 72 a segundo y 60 a tercero.

En la tabla 1 figur an las medias y las desviaciones típicas de las puntuaciones de la escala EASE para cada uno de los tres cursos y para el total de la muestra. Puede observarse que las mayor es puntuaciones corr esponden a los estudiantes de segundo curso. No obstante, las diferencias entre los tres cursos son escasas y el análisis de la varianza (ANOVA) determinó que estas diferencias no son estadísticamente significativas ( $F=0,886 ; p=0,414$ ). Tampoco los contrastes múltiples entre los cursos determinaron diferencias significativas en ninguno de los casos.

Tabla 1. Medias y desviaciones típicas de las puntuaciones de la EASE* para cada curso y para el total de la muestra

\begin{tabular}{lccc}
\hline Curso & N & media & Desv. Típica \\
Primero & 73 & 80,36 & 9,92 \\
Segundo & 72 & 82,14 & 7,34 \\
Tercero & 60 & 80,28 & 10,63 \\
Total & 205 & 80,96 & 9,32 \\
& & \\
*Escala de Actitud ante el Sida para Enfermería & \\
\hline
\end{tabular}

Con objeto de poder considerar la actitud de los alumnos ante cada uno de los enunciados propuestos, la tabla 2 contiene los porcentajes de respuestas directas a los ítems de la EASE para el total de la muestra. 
Tabla 2. Porcentaje de respuestas directas a cada una de las opciones de los ítems de la escala, para el total de la muestra $(\mathrm{N}=205)^{*}$

\begin{tabular}{l|c|c|c|c|c}
\hline Item & $\begin{array}{c}\text { Total } \\
\text { acuerdo }\end{array}$ & $\begin{array}{c}\text { Moderado } \\
\text { acuerdo }\end{array}$ & $\begin{array}{c}\text { Ni acuerdo } \\
\text { ni desacuerdo }\end{array}$ & $\begin{array}{c}\text { Moderado } \\
\text { desacuerdo }\end{array}$ & $\begin{array}{c}\text { Total } \\
\text { desacuerdo }\end{array}$ \\
\hline 1 & 1,1 & 1,5 & 0,4 & 3,4 & 93,6 \\
2 & 4,1 & 13,9 & 33,3 & 19,5 & 29,2 \\
3 & 69,7 & 18,4 & 3,7 & 4,9 & 3,4 \\
4 & 13,1 & 15,0 & 22,8 & 21,3 & 27,7 \\
5 & 81,6 & 7,5 & 2,6 & 3,4 & 4,9 \\
6 & 3,0 & 3,0 & 3,4 & 14,2 & 76,4 \\
7 & 57,7 & 20,2 & 5,6 & 9,4 & 7,1 \\
8 & 28,8 & 29,2 & 7,9 & 19,5 & 14,6 \\
9 & 6,4 & 8,2 & 4,1 & 18,0 & 63,3 \\
10 & 15,4 & 13,1 & 18,4 & 19,5 & 33,7 \\
11 & 80,5 & 9,0 & 0,7 & 3,7 & 6,0 \\
12 & 16,5 & 21,3 & 18,4 & 18,0 & 25,8 \\
13 & 27,3 & 29,6 & 18,7 & 13,9 & 10,5 \\
14 & 7,5 & 12,0 & 23,2 & 26,2 & 31,1 \\
15 & 35,6 & 22,5 & 12,7 & 16,9 & 12,4 \\
16 & 6,7 & 8,6 & 9,4 & 23,2 & 52,1 \\
17 & 4,1 & 7,1 & 11,2 & 18,4 & 59,2 \\
18 & 3,7 & 2,2 & 6,7 & 19,5 & 67,8 \\
19 & 6,7 & 4,9 & 4,9 & 22,5 & 61,0 \\
20 & 14,2 & 12,4 & 6,4 & 21,7 & 45,3 \\
21 & 55,4 & 16,9 & 9,0 & 10,5 & 8,2 \\
\hline
\end{tabular}

*Los enunciados de los ítems pueden verse en anexo

\section{DISCUSIÓN}

Del análisis de los resultados cabe destacar, en primer lugar, el hecho de que, en contra de lo esperado, no existen diferencias estadísticamente significativas entre los tres cursos de enfermería. Como puede verse en la tabla 1, las medias obtenidas en los cursos de primero, segundo y tercero, son prácticamente iguales y todas ellas superiores a la puntuación de 80. Con este resultado, a priori, podríamos conduir que la actitud de los estudiantes de enfermería ante los enfermos de SIDA es moderadamente favorable, teniendo en cuenta que la puntuación máxima, que representa la actitud más positiva, se sitúa en los 105 puntos.

Aunque, en principio, se había hipotetizado que el avance de curso, en cuanto supone un aumento de los conocimientos fisiopatológicos y humanistas, prácticas clínicas, madurez personal y otras experiencias individuales, se traduciría en un cambio hacia actitudes más positivas por parte de los alum- nos -hipótesis avalada por estudios previos ${ }^{29,30}$ - Io cierto es que los resultados no confirman este pre supuesto y resaltan una igualdad en cuanto a la actitud que no parece haber sido modificada por las variables mencionadas.

Por otro lado, aunque las puntuaciones globales de la escala parecen reflejar una actitud próxima a la que podría considerarse óptima para un futuro profesional de la salud, el análisis pormenorizado de las respuestas a cada uno de los ítems, contenidas en la tabla 2, nos da una visión menos optimista.

No obstante, antes de proceder a la interpretación de estas respuestas, es conveniente describir algunas características diferenciales entre los tres cursos. En el momento de responder a la escala, los estudiantes de primer curso han iniciado recientemente los estudios de enfermería, apenas han tenido ninguna dase de contenidos y por supuesto, no han tenido ningún contacto con la práctica dínica, lo que permite inferir que la actitud de este grupo no será muy diferente a la de la población general en su grupo de edad. Los alumnos de segundo curso, por su parte, han adquirido todos los conocimientos que se imparten en primero de carrera y han realizado prácticas clínicas durante un mes en diferentes instituciones sanitarias, aunque todavía no han adquirido conocimientos teóricos de fisiopatología sobre el VIH/SIDA. Los de tercer curso han hecho prácticas dínicas durante dos meses en diferentes hospitales y un mes en centros de atención primaria; además de haber cursado asignaturas teóricas donde se imparten conocimientos específicos sobre el tema.

Esta descripción de las características de los alumnos puede ayudarnos a una mejor interpretación y discusión de los resultados, especialmente de algunos ítems concretos.

En primer lugar, podemos destacar el hecho de que entre un 20 y un $25 \%$ de los estudiantes no tengan daro que debe guardarse el secreto profesional cuando hablamos de un portador del VIH (ítem 7). Así mismo, aproximadamente el 28\% también opinan que un enfermo con SIDA debe ser identificado como tal (item 10), resultados en la misma línea que los obtenidos en otros estudios ${ }^{31}$. Estos datos deberían hacernos reflexionar sobre el tipo de educación que están recibiendo nuestros estudiantes y sobre la necesidad de promover estrategias docentes que consigan una aproximación más humana en el cuidado de estos enfermos.

Por otro lado, también Ilama la atención que un $20 \%$ de los alumnos de primer y tercer curso opinen 
que, como medida de precaución, debería evitarse el contacto con los portadores del VIH y enfermos de SIDA (ítem 19), a la vez que casi un $40 \%$ del total de alumnos no tienen claro que en las actividades cotidianas no existe riesgo de contagio (ítem 8). E videntemente, esto conlleva la creencia que el riesgo aumentará considerablemente en el contacto directo con el paciente hospitalizado y propiciará el mantenimiento de serias precauciones en el contacto con estos enfermos.

Otro ítem que merece comentarse es el que hace referencia a la necesidad de llevar guantes siempre que se tenga contacto directo con un enfermo con SIDA (ítem 20). Un $20 \%$ de los alumnos de primero y segundo y un $40 \%$ de los de tercero están de acuerdo con la medida, de manera que se observa una progresión ascendente en relación con la creencia de la necesidad de la utilización de guantes conforme se avanza de curso. Una posible explicación a este hecho es la que apuntan otros estudios en los que se constata que el miedo al contagio aumenta con la realización de procedimientos invasivos ${ }^{32}$, dado que los alumnos de tercer curso son los que más prácticas clínicas han realizado y, por tanto, más técnicas invasivas han Ilevado a cabo. No obstante, también existen investigaciones que, alternativamente, sugieren que cuando los cuidados a enfermos de SIDA son más habituales se produce una falsa seguridad y se reducen las medidas universales de protección ${ }^{33}$.

En conclusión, los resultados obtenidos plantean la necesidad de una reflexión profunda acerca de las estrategias docentes más adecuadas que debemos utilizar en la modificación positiva de las actitudes y creencias que presentan los estudiantes ante el VIH/SIDA. Diferentes estudios han comprobado que la aplicación de programas específicos reducen la ansiedad y el miedo al contagio de las personas que cuidan a estos enfermos, a la vez que modifican los conocimientos sobre el tema ${ }^{13,3436}$.

\section{BIBLIOGRAFÍA}

1. Wang J F, Paterson J. Using factor analysis to explore nurses' fear of AIDS in the United States of America. J Adv Nurs 1996; 24: 287-295.

2. Ventura MJ. Ethics on the job: a survey. The realities of HIV/AIDS. R N 1999; 62(4): 26-30.

3. Ward C. Caring by degrees. Contemp Nurse 1998; 7(1): 24-28.

4. Bartnof HS. Health care professional education and AIDS. Death Stud 1988; 12(5-6): 547-562.

5. Robinson N. People with HIV/AIDS: who cares? J Adv Nurs 1998; 28(4): 771-778.
6. Green G, Platt S. Fear and loathing in health care settings reported by people with HIV. Soc Health IIIness 1997; 19: 7092.

7. Steele A, Melby V. Nurses' knowledge and beliefs about AIDS: comparing nurses in hospital, community and hospice settings. J Adv Nurs 1995; 22: 879-887.

8. Cole FL. Factors Associated with Student Nurses' intent to provide physical and psychosocial care to persons with Acquired Immunodeficiency Syndrome. J Prof Nurs 1996; 12(4): 217-224.

9. West AM, Leasure R, Allen P, Lagrow P. Attitudes of baccalaureate nursing students toward persons with acquired immunodeficiency syndrome according to mode of human immunodeficiency virus transmission. J Prof Nurs 1996; 12(4): 225-232.

10. Strasser JA, Damrosch S. Graduate Nursing Students' attitudes toward gay and hemophiliac men with AIDS. Eval Health Prof 1992; 15(1): 15-27.

11. Leasure R, McKenney LA, Merrill A. Factors influencing Baccalaureate nursing students' attitudes toward persons living with AIDS. J Prof Nurs 1995; 11(5): 299-305.

12. Niven CA, Knussen $C$. Measuring the stress associated with caring for dients with HIV/AIDS. AIDS Care 1999; 11(2): 171180.

13. Bennett J. Fear of contagion: a response to stress? ANS Adv Nurs Sci 1998; 21(1): 76-87.

14. Peate I, Souminen T, Valimaki M, Lohrmann C, Muinonen U. HIV/AIDS and its impact on student nurses. Nurse Educ Today 2002; 22(6): 492.

15. Swanson J M, Chenitz C, Zalar M, Stoll P. A critical review of human immunodeficiency virus infection and acquired immunodeficiency syndrome related research: The knowledge, attitudes, and practice of nurses.J Prof Nurs 1990; 6(6): 341-355.

16. Ficarrotto TJ , Grade M, Bliwise N, I sish T. Predictors of medical and nursing students' levels of AIDS knowledge and their resistance to working with AIDS- HIV patients. Acad Med 1990; 65(7): 470-471.

17. Välimäki M, Souminen T, Peace I. Attitudes of professionals, students and the general public to HIV/AIDS and people with HIV/AIDS: a review of the research. J Adv Nurs 1998; 27(4): 752-759.

18. Webb AA, Bower DA, Gill S. Satisfaction with nursing care: a comparison of patients with HIV/AIDS, non-HIV/AIDS infectious diseases, and medical diagnoses. J Assoc Nurses AIDS Care 1997; 8(2): 39-46.

19. Tabak N, Ben Or T, Zur F. Health nurses: attitudes towards treating AIDS patients. Med Law 1997; 16(4): 729-742.

20. Wyness MA Goldstone I. Teaching about HIV/AIDS: the lessons learned. Int Nurs rev 1998; 45(6): 182-186.

21. Tesch BJ, Simpson DE, Kirby BD. Medical and Nursing Students' attitudes about AIDS I ssues. Acad Med 1990; 65(7): 467-469.

22. Chacartegui Martínez I. SIDA, Enfermedad profesional. Enferm Intensiva 1992; 3(2): 54-56.

23. Wiley K, Heath L, Adklin M. Care of AIDS patients: Student attitudes. Nurs Outlook 1988; 36(5): 244-245.

24. Currey CJ , J ohnson M, Ogden B. Willingness of health professions students to treat patients with AIDS. Acad Med 1990; 65(7): 472-474. 
25. Bennett JA. Nurses' attitudes about acquired immunodeficiency syndrome care: What research tells us. J Prof Nurs 1995; 11(6): 339-350.

26. Meisenhelder J B. Contributing factors to fear of HIV contagion in registered nurses. Image J Nurs Sch 1994; 26(1): 6569.

27. Grossman S, Wheeler K, Lippman D. Role-modeling experience improves nursing students' attitudes toward people living with AIDS. Nursingconections 1998; 11(1): 41-49.

28. Tomás-Sábado J . Actitud de enfermería ante el SIDA; construcción de una escala de Likert. Enferm Clínica 1999; 9(6): 233-237.

29. Snowden L. An investigation into whether nursing student al ter their attitudes and knowl edge levels regarding HIV infection and AIDS fol lowing a 3-year programme leading to registration as a qualified nurse. J adv nurs 1997; 25(6): 1167-1174.

30. West AM, Davis-Lagrow P, L easure R, Allen P. L ow versus high prevalence of AIDS: effect on nursing students' attitudes and knowl edge. AIDS Patient Care STDS 1998; 12(1): 51-60.
31. Lal P, Kumar A, Ingle GK, Gulati N. Some AIDS-related policy issues and nursing students' willingness to provide AIDS care. J Commun Dis 1998; 30(1): 38-43.

32. McCann TV, Sharkey RJ. Educational intervention with international nurses and changes in knowledge, attitudes and willingness to provide care to patients with HIV/AIDS. J AdV Nurs 1998; 27(2): 267-273.

33. Grellier R. Everyone is scared of it inside so they start being a bit irrational': HIV/AIDS education within midwifery. Midwifery 2000; 16(1): 56-67.

34. All AC, Sullivan L. The effects of an HIV/Aids educational programme on the anxiety level of nursing students. J Adv Nurs 1997; 26(4): 798-803.

35. Valois P, Turgeon H, Godin G, Blondeau D, Cote F. Influence of a persuasive strategy on nursing students' beliefs and attitudes toward provision of care to people living HIV/AIDS. J Nurs Educ 2001; 40(8): 354-358.

36. Tomás-Sábado J, Aradilla A. Educación sobre la muerte en estudiantes de enfermería. Eficacia de la metodología experiencial. Enferm Científica 2001; 234-235: 65-72.

\section{Anexo. Items de la Escala de Actitud ante el SIDA para enfermería}

1. El SIDA no afecta a las parejas heterosexuales.

2. Los fetos contagiados con el virus del SIDA deberían abortarse.

3. No existe ningún peligro en el hecho de que los portadores del virus del SIDA utilicen restaurantes y bares públicos.

4. No debe permitirse que las mujeres seropositivas queden embarazadas.

5. El SIDA es un problema de todos.

6. La atención continuada a un enfermo de SIDA es sinónimo de contagio.

7. El por tador del virus del SIDA tiene derecho a que se guarde el secreto médico de su diagnóstico.

8. En las actividades cotidianas no hay ningún riesgo de transmisión del virus del SIDA.

9. Los enfermos de SIDA deben ser aislados del resto de enfermos.

10. El seropositivo debe ser identificado como tal.

11. Ser portador del virus del SIDA no debe ser obstáculo para acceder a la educación y el empleo.

12. Deberían crearse hospitales específicos para enfermos de SIDA y portadores.

13. El SIDA es la mayor plaga de nuestro tiempo.

14. El enfermo de SIDA debe ser considerado una víctima del sistema social.

15. El ser portador del virus del SIDA no debería ser impedimento para poder adoptar un niño.

16. En el hospital, un portador del virus del SIDA no debería compartir habitación con una persona no infectada.

17. No me resultaría agradable trabajar con un compañero portador del virus del SIDA.

18. Los niños portadores del virus del SIDA deberían acudir a clases especiales.

19. Como medida de precaución debemos evitar el contacto con los enfermos de SIDA y portadores.

20. Siempre que toquemos a un enfermo de SIDA debemos utilizar guantes.

21. La prueba del SIDA debe ser voluntaria y anónima. 